\title{
Clinical value of miRNA-122 in the diagnosis and prognosis of various types of cancer
}

\author{
MEIYU DAI, LIMIN LI and XUE QIN \\ Department of Clinical Laboratory, First Affiliated Hospital of Guangxi Medical University, \\ Nanning, Guangxi 530021, P.R. China
}

Received January 12, 2018; Accepted February 6, 2019

DOI: $10.3892 / \mathrm{ol} .2019 .10024$

\begin{abstract}
The present study aimed to systematically analyze the value of microRNA-122 (miRNA-122) in the diagnosis and prognosis of hepatocellular carcinoma (HCC) and other types of cancer. First, the reverse transcription-quantitative polymerase chain reaction method was used to detect the expression levels of miRNA-122 in the serum samples of patients with HCC, benign lesions and healthy volunteers. Next, miRNA-seq data of miRNA-122 from The Cancer Genome Atlas database were used to analyze the differential expression and overall survival rate associated with a variety of types of cancer. Meanwhile, the target gene prediction of miRNA-122 was performed using four different software programs. Finally, 353 significant target genes were identified for Gene Ontology and Kyoto Encyclopedia of Genes and Genomes functional enrichment analysis. Finally, it was demonstrated that the expression levels of miRNA-122 in the HCC group were increased compared with the healthy group $(\mathrm{P}<0.001)$, but decreased with respect to the benign group $(\mathrm{P}<0.001)$. In addition, the combination of the miRNA-122 and a fetoprotein may further improve the diagnostic accuracy between the HCC and healthy groups (area under the curve, 0.980; 95\% confidence interval, 0.958-1.000). It was also demonstrated that miRNA-122 exhibited significantly differential expression and the overall survival rate was predicted for various other types of cancer, including colorectal cancer, renal carcinoma, cholangiocarcinoma, prostate cancer and thyroid carcinoma. Functional enrichment analysis demonstrated that the target genes of miRNA-122 may contribute to the composition of the nucleus and cytoplasm, and regulate a variety of biological processes, including cardiac muscle cell differentiation and glucose metabolic processes via protein biosynthesis, estrogen and glucagon associated signaling pathways. These results
\end{abstract}

Correspondence to: Professor Xue Qin, Department of Clinical Laboratory, First Affiliated Hospital of Guangxi Medical University, 6 Shuangyong Road, Nanning, Guangxi 530021, P.R. China E-mail: qinxue919@126.com

Key words: microRNA-122, cancer, The Cancer Genome Atlas, diagnosis, overall survival rate revealed that miRNA-122 may be an indispensable biomarker for the diagnosis, prognostic evaluation and targeted therapy in pan-cancer.

\section{Introduction}

Hepatocellular carcinoma (HCC) is the sixth most common form of cancer (1) and is the third most common cause of cancer-associated mortality worldwide (2). Hepatitis B virus (HBV) infection is one of the important etiological factors that can lead to liver damage. It was estimated that $\sim 5 \%$ of the world population are infected with $\mathrm{HBV}$, especially in China (3). A large proportion of patients with chronic HBV infection may develop necroinflammatory liver disease in the order of increasing severity from sustained liver injury to cirrhosis, hepatic failure and HCC (4). Early diagnosis and treatment are vital for improving the overall survival rate of patients with HCC. The detection of serum a fetoprotein (AFP) may aid the diagnosis of HCC, but its sensitivity is low, especially at the early stage of HCC. Therefore, it is critical to find novel non-invasive serum biomarkers for the independent or combined diagnosis of HCC.

MicroRNA (miRNA), a type of small non-coding single stranded RNA of 18-25 nucleotides in length, can interact with the target genes to suppress their expression by promoting the degradation of target genes or inhibiting translation $(5,6)$. miRNAs have a wide range of functions and are involved in a variety of biological processes, including cell proliferation, growth, differentiation, apoptosis, metabolism and signaling pathway transduction $(7,8)$. They may also serve as important clinical biomarkers for the diagnosis and prognosis of multiple types of cancer (9-11). miRNA-122 is one of the key biomarkers of the miRNAs and constitutes $\sim 52 \%$ of the total hepatic miRNome (12). It is the most abundant liver-specific miRNA, which has notable anti-inflammatory effects and is involved in a variety of liver functions $(13,14)$. It has been reported that miRNA-122 exhibited dysregulated expression in HCC tissues, serum and cell lines $(15,16)$; however, contradictory conclusions regarding the differential expression and prognosis of miRNA-122 have been reported (17). In addition, the numerous functions of miRNA-122 in HCC and other types of cancer remain to be further investigated.

In the present study, the clinical value and associated function of miRNA-122 in various types of cancer, particularly 
in HCC were systematically analyzed. Firstly, the expression levels of miRNA-122 in patients with HCC, benign and healthy groups were analyzed and the significance of miRNA-122 and AFP in the differential diagnosis of HCC was evaluated. Secondly, the analysis on the differential expression and survival rate of miRNA-122 in a variety of common cancer types using miRNA-seq data from The Cancer Genome Atlas (TCGA; https://cancergenome.nih.gov/) was performed. Thirdly, the target genes of miRNA-122 were comprehensively predicted for further functional enrichment analysis. Finally, the associated research progress of miRNA-122 was systematically summarized. The content of the present study was progressive and may improve current understanding of miRNA-122 from a macroscopic and comprehensive perspective.

\section{Materials and methods}

Serum sample collection. The present study was approved by the Institutional Review Board of the First Affiliated Hospital of Guangxi Medical University (Guangxi, China) and written informed consent was obtained from all individuals in this research project. A total of 150 individuals were enrolled from September 2016 to September 2017, including patients with HCC (50 cases), patients with benign lesions (50 cases) and healthy controls (50 cases). Patent characteristics are presented in Table I. HCC patients were diagnosed by serum AFP concentration analysis and abdominal ultrasonography; diagnoses were confirmed by histopathological examination. In addition, all the serum samples of HCC patients were collected prior to surgical resection. All the serum samples were collected, centrifuged at $2,000 \mathrm{x} \mathrm{g}$ for $5 \mathrm{~min}$ at room temperature and stored as $500-\mu 1$ aliquots at $-80^{\circ} \mathrm{C}$ prior to experimental use.

Reverse transcription-quantitative polymerase chain reaction (RT-qPCR) experiment. Total RNA of serum samples was extracted using the Serum/Plasma miRNA Extraction and Isolation kit DP503. This kit is a newly-developed products specifically for serum and plasma miRNA extraction. Total RNA was reverse transcribed using the miRcute Enhanced miRNA cDNA First Strand Synthesis kit (KR211). All the reagents were obtained from Tiangen Biotech Co., Ltd. (Beijing, China) and all operations were conducted in strict accordance with the manufacturer's protocol. The temperature protocol was as follows: $60 \mathrm{~min}$ for miRNA plus A tail reaction and reverse transcription reaction at $42^{\circ} \mathrm{C} ; 3 \mathrm{~min}$ for enzyme inactivation reaction at $95^{\circ} \mathrm{C}$. The RT-qPCR experiment was performed using the reagent of SYBR-Green FP411. The thermocycling conditions of the RT-qPCR were as follows: $15 \mathrm{~min}$ for initial template denaturation at $95^{\circ} \mathrm{C}$, following 45 cycles of PCR template denaturation for $20 \mathrm{sec}$ at $94^{\circ} \mathrm{C}$; annealing and extension for $34 \mathrm{sec}$ at $60^{\circ} \mathrm{C}$. The melting/dissociation curve stage was produced following RT-qPCR. The 7500 Real-Time PCR Instrument from Thermo Fisher Scientific, Inc. (Waltham, MA, USA) was used in the detection. U6 was set as an internal reference. The primer of U6: Forward: 5'-AGCGGGAAATCG TGCGTGACA-3', reverse: 5'-GTGGACTTGGGAGAGGAC TGG-3'. The primer of miRNA-122: Forward: 5'-GCGAAA GCATTTGCCAAGAA-3', reverse: 5'-CATCACAGACCT GTTATTGC-3'. Calculation of the relative miRNA expression levels was based on $2^{-\Delta \Delta \mathrm{Cq}}(18)$.
Statistical analysis. The values in tables were reported as the mean \pm standard deviation or number percentage, unless otherwise stated. The receiver operating characteristic curve, area under curve (AUC), sensitivity, specificity, positive predictive value and negative predictive value (NPV) were used to analyze diagnostic accuracy. Youden index=sensitivity-(1-specificity). When the Youden index is at its maximum, the corresponding detection value can be used as the cutoff (19). Student's t-test or one-way analysis of variance (ANOVA) was used to analyze continuous variables where appropriate in this study. Student's t-test was used for the analysis of differential expression between two groups and the one-way ANOVA was used for the analysis of differential expression among more than two groups. Bonferroni's post hoc test was used following the ANOVA. The $\chi^{2}$ test or Fisher's exact test was used for the comparisons of differences in the categorical data between groups. A two-tailed $\mathrm{P} \leq 0.05$ was considered to indicate a statistically significant difference. The aforementioned analyses were performed using IBM SPSS 20.0 (IBM, Corps., Armonk, NY, USA). The scatter plot was produced using GraphPad Prism 5 software (Graphpad Software, Inc., La Jolla, CA, USA).

Differential expression and survival analysis of miRNA-122 in TCGA and literature summary in PubMed. At present, a number of studies have demonstrated that miRNAs or other genes frequently exhibit diversity as clinical markers of cancer. The diagnosis and prognosis of a certain biomarker in clinical pan-cancer is also a popular research model $(20,21)$. Therefore, data of TCGA was thoroughly used to analyze the expression levels of miRNA-122 in a variety of common tumors in this study. Data mining is one of the hot topics in bioinformatics research. The statistical results are more scientific and accurate than simple difference analysis. TCGA is currently a popular database for cancer research. It is a project initiated by the National Cancer Institute and the National Human Genome Research Institute in 2005. It has provided a large amount of genomic data and associated clinical data for researchers in basic and translational medicine for cancer. In this study, analysis of differential expression was performed on the basis that cancer tissues and para-cancerous tissues must be present simultaneously. In TCGA database, the number of tumor tissues are greater than the normal tissues. Certain types of tumor do not contain normal tissue samples. Therefore, the criteria that adjacent normal tissue samples $\geq 3$ was set. The 'edgeR' package of R (version 3.3.3; https://www.r-project.org) was used for differential expression analysis based on a False Discovery Rate $<0.05$ and the absolute value of $\log _{2}$ (fold change) $\geq 1$. In addition, the overall survival analysis of cancers with differentially expressed miRNA-122 was performed using the 'survival' package of $\mathrm{R}$ (http://bioconductor. org/packages/3.6/bioc/). The present study also searched for relevant references by searching the keyword 'miRNA-122' or 'microRNA-122' or 'miR-122' in PubMed (https://www. ncbi.nlm.nih.gov/pubmed), and summarized the expression and prognosis of miRNA-122 in various types of cancer.

Target gene prediction and functional enrichment analysis. The target gene prediction software available for miRNA is 
Table I. Clinical characteristics of the included individuals.

\begin{tabular}{|c|c|c|c|c|c|}
\hline Characteristics & $\mathrm{HCC}$ & Hepatitis B & Cirrhosis & Healthy & P-value \\
\hline Mean age $\pm S D$, years & $48.6 \pm 11.9$ & $42.9 \pm 14.4$ & $48.0 \pm 11.0$ & $47.2 \pm 11.7$ & $>0.05$ \\
\hline \multicolumn{6}{|l|}{ Sex } \\
\hline Male, n (\%) & $35(70)$ & $17(68)$ & $18(72)$ & $35(70)$ & $>0.05$ \\
\hline Female, n (\%) & $15(30)$ & $8(32)$ & $7(28)$ & $15(30)$ & \\
\hline AFP, ng/ml & $10,676.3 \pm 20,568.7$ & $32.4 \pm 74.3$ & $20.5 \pm 47.5$ & $2.7 \pm 1.6$ & $<0.001$ \\
\hline \multicolumn{6}{|l|}{ Cirrhosis } \\
\hline Yes, n (\%) & $38(76)$ & $0(0)$ & $25(100)$ & $0(0)$ & \\
\hline No, n (\%) & $12(24)$ & $25(100)$ & $0(0)$ & $50(100)$ & \\
\hline \multicolumn{6}{|l|}{ Hepatitis B } \\
\hline Yes, n (\%) & $42(84)$ & $25(100)$ & $7(28)$ & $0(0)$ & \\
\hline No, n (\%) & $8(16)$ & $0(0)$ & $18(72)$ & $50(100)$ & \\
\hline
\end{tabular}

Sample sizes in different groups: HCC, $n=50$; hepatitis B, $n=25$; cirrhosis, $n=25$; healthy, $n=50$. The $P$-value comparing the difference between the sexes was calculated using $\chi^{2}$ test; the P-value comparing the differences between ages and AFP was calculated using a one-way analysis of variance statistical method depending on data normality. HCC, hepatocellular carcinoma; AFP, $\alpha$-fetoprotein.
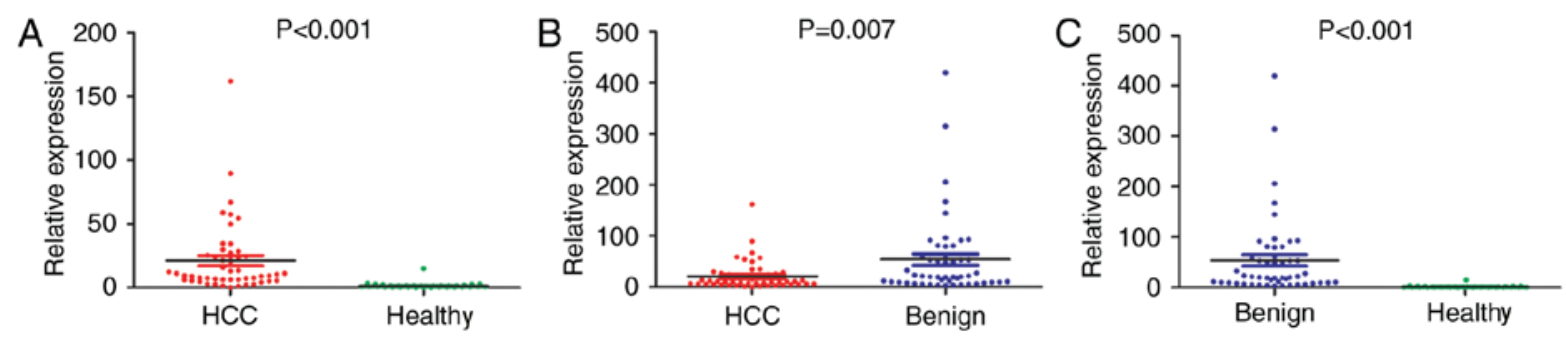

Figure 1. Expression levels of miRNA-122 in serum samples in HCC, benign and healthy groups. (A) The HCC group vs. the Healthy group. (B) The HCC group vs. the Benign group. (C) The Benign group vs. the Healthy group. The data conforms to normal distribution and a one-way analysis of variance method was used. HCC, hepatocellular carcinoma; miR, microRNA.

multifarious. In the present study, four classical and common sets of software were selected to predict the target gene of miRNA-122, including miRanda (http://www.microrna. org/) (22), Pic Tar (http://pictar.mdc-berlin.de/) (23), miRDB (http://www.mirdb.org/) (24) and TargetScanHuman (http://www.targetscan.org/vert_71/) (25), respectively. Comparing the target genes of the four types of software, the intersection of any two predictor softwares were selected as candidate target genes for Gene Ontology (GO) and Kyoto Encyclopedia of Genes and Genomes (KEGG) pathway functional enrichment analyses. The Venn diagram demonstrating intersections were constructed using the 'VennDiagram' package of R. Furthermore, the GO and KEGG pathway analyses are the most common functional enrichment analyses and were performed using the online software, Database for Annotation, Visualization and Integrated Discovery (DAVID), version 6.8 (https://david.ncifcrf.gov/) (26). The selection criteria for functional items were in accordance with $\mathrm{P} \leq 0.05$ and count number $\geq 2$.

\section{Results}

Clinical characteristics. The clinical information and laboratory biomarker analysis of the different groups are presented in Table I. For statistical analysis, all the participants were divided into three groups: Including HCC (50 cases), benign (HBV and cirrhosis, 50 cases) and healthy (50 cases). There was no statistically significant difference between the age and gender of the studied groups as they were matched with each other. However, there was a significant difference between the different groups regarding the clinical biomarker AFP ( $\mathrm{P}<0.001$ for all comparisons).

Different expression of miRNA-122 among three groups. The expression levels of miRNA-122 were detected among the HCC, benign and healthy groups. The results indicated that there was a significant difference in the expression levels of miRNA-122 among the three groups (Fig. 1). Compared with the healthy group, the HCC and benign groups exhibited significantly increased expression levels of miRNA-122 $(\mathrm{P}<0.001)$. In addition, the expression levels of miRNA-122 between the HCC and benign groups were compared, and it was demonstrated that the benign group exhibited significantly higher expression levels of miRNA-122 compared with the HCC group $(\mathrm{P}=0.007)$. Overall, the expression levels of miRNA-122 in the healthy, HCC and benign groups demonstrated a stepwise increase (healthy group $<$ HCC group < benign group); there was a statistically significant difference between the 
groups ( $\mathrm{P}<0.001$; Fig. 1C). Therefore, miRNA-122 can be used not only for the identification of HCC and normal controls, but also for the identification of HCC and benign lesions. There is also a study demonstrating that the serum level of miRNA-122 in patients with hepatitis is increased compared with in HCC patients (27). The experiments of the present study confirmed this.

Subgroup analysis of miRNA-122 in individuals with HBV. In the study of the clinical markers of HCC, it is particularly important to analyze individuals with hepatitis B. Therefore, subgroup analysis (HBV-positive and -negative groups) of the expression levels in individuals with hepatitis B was performed in the present study. The results are presented in Table II. There was no statistically significant difference in the expression levels of miRNA-122 between the positive and negative HBV groups. However, the concentration of AFP was significantly associated with HBV infection in the HCC $(\mathrm{P}=0.03)$ and $\mathrm{HCC}+$ Benign group $(\mathrm{P}=0.003)$. In $\mathrm{HBV}$-infected individuals, AFP concentrations were increased compared with HBV negative patients.

Diagnostic accuracy of miRNA-122, AFP and miRNA-122 + AFP. In the previous differential expression analysis, it was demonstrated that the miRNA-122 expression levels were significantly different between the HCC vs. healthy groups and the HCC vs. benign groups (Fig. 1). Additionally, AFP has also been recognized as a serological biomarker for the diagnosis of HCC. Therefore, the diagnostic value of assessing miRNA-122, AFP and miRNA-122 + AFP in HCC was investigated in the present study (Table III). The diagnostic sensitivity of miRNA-122 (sensitivity, 90\%) was increased compared with AFP (sensitivity, 86\%) and the combination of the two biomarkers could further improve the diagnostic accuracy between the HCC and healthy groups (AUC, 0.980; 95\% CI, 0.958-1.000). However, the diagnostic sensitivity and specificity of miRNA-122 were poor between HCC and benign groups (sensitivity, 64\%; specificity, 62\%).

Differential expression and survival analysis of miRNA-122 by pan-cancer analysis of TCGA database. It is an indisputable fact that miRNA-122 is highly expressed in HCC. However, the expression of miRNA-122 in other types of tumor requires further investigation. In the present study, the differential expression of miRNA-122 was analyzed in a variety of common tumors using the publicly available data of TCGA database. Following strict screening, 17 types of cancer were selected to analyze the differential expression of miRNA-122 between cancerous and adjacent normal tissues; the results are presented in Table IV. The expression levels of miRNA-122 were significantly upregulated in eight types of cancer, including colorectal carcinoma, breast, kidney and lung cancers, prostate adenocarcinoma, stomach adenocarcinoma, thyroid carcinoma and uterine cancer. In addition, miRNA-122 was reported to be significantly downregulated in cholangiocarcinoma and HCC (Table IV). Simultaneously, survival analysis was performed in the types of cancer with differentially expressed miRNA-122. The results of survival analysis demonstrated that high expression levels of miRNA-122 were associated with poor prognosis in kidney 
Table III. Diagnostic value of miRNA-122, AFP and miRNA-122 + AFP.

\begin{tabular}{|c|c|c|c|c|c|c|}
\hline \multirow[b]{2}{*}{ Parameter } & \multicolumn{3}{|c|}{ HCC vs. Healthy } & \multicolumn{3}{|c|}{ HCC vs. Benign } \\
\hline & $\operatorname{miR}-122$ & AFP & $\mathrm{miR}-122+\mathrm{AFP}$ & $\operatorname{miR}-122$ & AFP & miR-122 + AFP \\
\hline Sensitivity, \% & 90.0 & 86.0 & 90.0 & 64.0 & 86.0 & 86.0 \\
\hline Specificity, \% & 94.0 & 100.0 & 100.0 & 62.0 & 80.0 & 80.0 \\
\hline AUC $(95 \% \mathrm{CI})$ & $\begin{array}{c}0.954 \\
(0.907-1.000)\end{array}$ & $\begin{array}{c}0.961 \\
(0.927-0.996)\end{array}$ & $\begin{array}{c}0.980 \\
(0.958-1.000)\end{array}$ & $\begin{array}{c}0.667 \\
(0.562-0.772)\end{array}$ & $\begin{array}{c}0.878 \\
(0.811-0.944)\end{array}$ & $\begin{array}{c}0.876 \\
(0.808-0.944)\end{array}$ \\
\hline PPV, \% & 94.0 & 1.0 & 1.0 & 63.0 & 81.0 & 81.0 \\
\hline NPV, \% & 90.0 & 88.0 & 91.0 & 63.0 & 85.0 & 85.0 \\
\hline Cutoff & 2.80 & 8.78 & & 14.29 & 8.59 & \\
\hline
\end{tabular}

HCC, hepatocellular carcinoma; AFP, $\alpha$-fetoprotein; miR, microRNA; AUC, area under curve; PPV, positive predictive value; NPV, negative predictive value.

Table IV. Differential expression analysis of microRNA-122 in 17 types of cancer from The Cancer Genome Atlas database.

\begin{tabular}{|c|c|c|c|c|}
\hline Cancer type & Abbreviation & Cancer/normal, $\mathrm{n}$ & False discovery rate & $\operatorname{LogFC}$ \\
\hline Colorectal carcinoma & $\mathrm{CRC}$ & $619 / 11$ & $4.62 \times 10^{-2 \mathrm{a}}$ & 8.18 \\
\hline Adrenal gland carcinoma & $\mathrm{AGC}$ & $264 / 3$ & 1.00 & 7.02 \\
\hline Cholangiocarcinoma & $\mathrm{CHOL}$ & $36 / 9$ & $5.01 \times 10^{-8 a}$ & -4.16 \\
\hline Bladder urothelial carcinoma & BLCA & $418 / 19$ & $3.59 \times 10^{-1}$ & 2.04 \\
\hline Brain glioma & $\mathrm{BG}$ & $530 / 5$ & 1.00 & 0.45 \\
\hline Breast cancer & $\mathrm{BC}$ & $1,103 / 104$ & $1.80 \times 10^{-13 a}$ & 7.34 \\
\hline Cervical cancer & $\mathrm{CC}$ & $309 / 3$ & 1.00 & 6.52 \\
\hline Esophageal carcinoma & ESCA & $187 / 13$ & $2.53 \times 10^{-1}$ & 3.00 \\
\hline Head and neck squamous cell carcinoma & HNSC & $525 / 44$ & $7.73 \times 10^{-2}$ & -0.81 \\
\hline Kidney cancer & $\mathrm{KC}$ & $1,074 / 147$ & $9.40 \times 10^{-36 a}$ & 5.43 \\
\hline Hepatocellular carcinoma & $\mathrm{HCC}$ & $375 / 50$ & $1.14 \times 10^{-3 a}$ & -0.61 \\
\hline Lung cancer & $\mathrm{LC}$ & $999 / 91$ & $9.40 \times 10^{-11 a}$ & 5.75 \\
\hline Pancreatic adenocarcinoma & PAAD & $179 / 4$ & 1.00 & 0.35 \\
\hline Prostate adenocarcinoma & PRAD & $499 / 52$ & $1.19 \times 10^{-6 a}$ & 3.84 \\
\hline Stomach adenocarcinoma & STAD & $446 / 45$ & $9.86 \times 10^{-4 a}$ & 3.44 \\
\hline Thyroid carcinoma & THCA & $514 / 59$ & $7.77 \times 10^{-3 a}$ & 1.64 \\
\hline Uterine cancer & $\mathrm{UC}$ & $603 / 33$ & $3.28 \times 10^{-5 a}$ & 6.90 \\
\hline
\end{tabular}

${ }^{\mathrm{a}} \mathrm{P}<0.05 . \mathrm{LogFC}, \log _{2}$ (fold-change).

cancer, but good in patients with $\mathrm{HCC}(\mathrm{P}=0.012$ and $\mathrm{P}<0.001)$. However, there was no significant effects on the prognoses of other types of cancer (Fig. 2).

Literature summary of the expression and prognosis of miRNA-122 via pan-cancer analysis. In addition to experimental validation and public database mining, the collective findings of the literature were also valuable. Information from available articles on the PubMed database on differential expression levels and prognostic value of miRNA-122 in a variety of cancer types was summarized. The results are presented in Table V. Literature data of the differential expression and survival analysis of miRNA-122 between HCC and adjacent normal tissues were consistent with the results of TCGA analysis. Additionally, the results in colorectal cancer, renal carcinoma, cholangiocarcinoma, prostate cancer and thyroid carcinoma were the same. However, there were inconsistent conclusions of particular types of cancer, including breast and gastric cancer. The inconsistencies need to be further studied.

Target gene prediction of miRNA-122 and functional enrichment analysis. Due to the limitations of using a single prediction software of miRNA target genes, four commonly used miRNA target gene prediction software were used to simultaneously analyze. The intersection of two or more of the employed software was selected. Finally, 353 miRNA-122 target genes were included for functional enrichment analysis (Fig. 3). MEF2C, MASP1, SLC52A2, NONO, BRPF1, USP53, VPS4A, CCAR1, 
Table V. Differential expression and prognosis evaluation of miRNA-122 in various types of cancer from the reported literature.

\begin{tabular}{|c|c|c|c|c|}
\hline Tumor type & Sample type & $\begin{array}{l}\text { Differential expression } \\
\text { (cancer vs. normal) }\end{array}$ & Overall survival & (Refs.) \\
\hline Hepatocellular carcinoma & Tissue & Downregulated & Poor & (52) \\
\hline Hepatocellular carcinoma & Plasma/serum & Upregulated & Poor & $(57,58)$ \\
\hline Hepatocellular carcinoma & Plasma/serum & & Better & $(59,60)$ \\
\hline Hepatocellular carcinoma & Plasma/serum & Downregulated & & $(27)$ \\
\hline Hepatocellular carcinoma & Plasma/serum & No significance & & $(49,63)$ \\
\hline Glioma & Tissue & Downregulated & & $(32,64)$ \\
\hline Glioma & Plasma & Downregulated & Poor & (54) \\
\hline Lymphoma & Plasma & Downregulated & & $(65)$ \\
\hline Renal cell carcinoma & tissue & Upregulated & & $(33,66-71)$ \\
\hline Gastric cancer & Tissue & Downregulated & & \\
\hline Gastric cancer & Plasma & Downregulated & Poor & (55) \\
\hline Pancreatic cancer & Tissue & Downregulated & & $(35,72)$ \\
\hline Pancreatic cancer & Whole blood & Upregulated & & (73) \\
\hline Colorectal carcinoma & Tissue & Upregulated & & (53) \\
\hline Colorectal carcinoma & Plasma & Upregulated & & (74) \\
\hline Cholangiocarcinoma & Tissue & Downregulated & & (75) \\
\hline Gallbladder cancer & Tissue & Downregulated & & (76) \\
\hline Prostate cancer & Tissue & Upregulated & & (77) \\
\hline Retinoblastoma & Tissue & Downregulated & & (78) \\
\hline Osteosarcoma & Tissue & Downregulated & & (79) \\
\hline Breast cancer & Tissue & Downregulated & & $(80-82)$ \\
\hline Papillary thyroid carcinoma & Tissue & Upregulated & & (83) \\
\hline Pituitary carcinomas & Tissue & Upregulated & & (84) \\
\hline Acute myeloid leukemia & Tissue & Downregulated & Poor & (56) \\
\hline Bladder cancer & Tissue & Downregulated & & $(85)$ \\
\hline
\end{tabular}

A

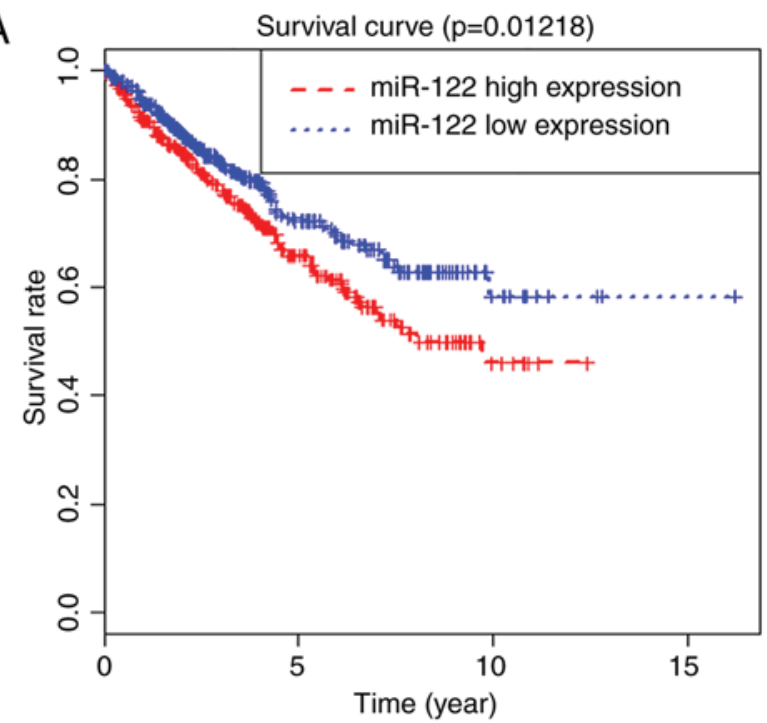

B

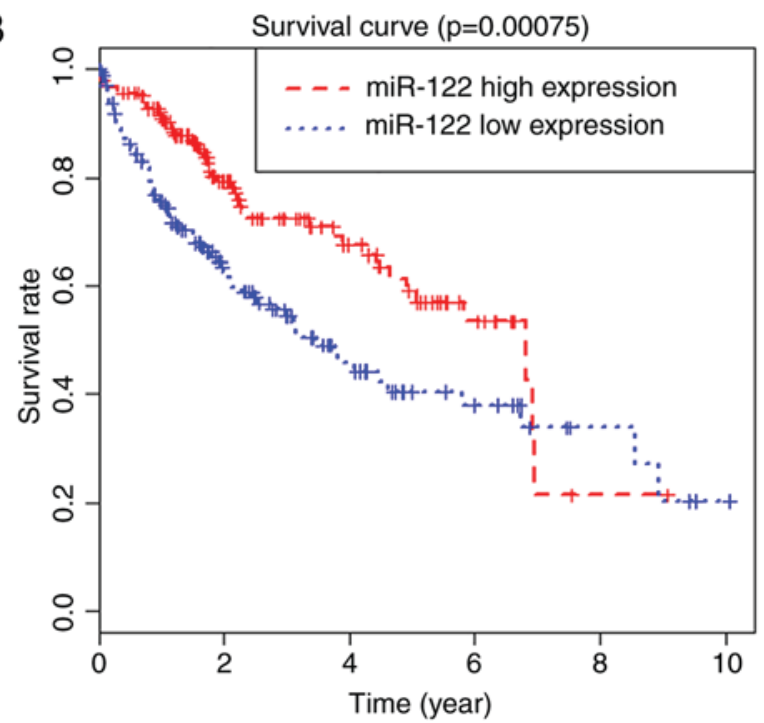

Figure 2. Survival curve of miRNA-122 in kidney cancer and HCC. (A) miRNA-122 low expression indicated a higher overall survival rate in kidney cancer. (B) miRNA-122 high expression indicated higher overall survival rate in HCC. Kaplan-Meier and log-rank test were used for survival analysis. HCC, hepatocellular carcinoma; miR, microRNA.

H1F0 and KIF5B were the top 10 of the 353 target genes, and the others are not illustrated. In the present study, GO and
KEGG pathway analysis were carried on the target genes of miRNA-122. Following the enrichment analysis by DAVID 


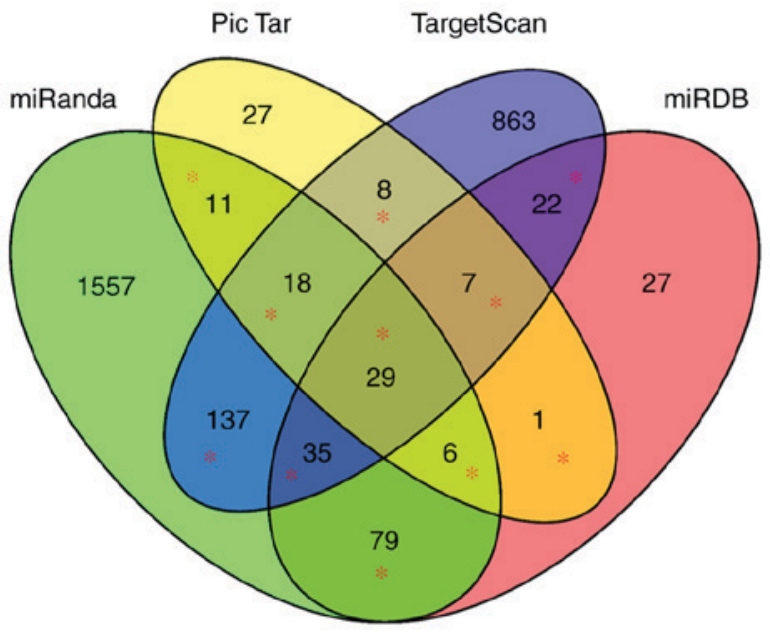

Figure 3. Venn diagram of four different software predictions for the target genes of microRNA-122. The area with the red asterisk in the figure is the included target genes, with a total of 353 target genes.

software, 68 notable GO items were identified, of which 38 items for 'biological process' (BP), 8 items for 'cellular component' (CC) and 22 items for 'molecular function' (MF). In addition, six important KEGG signaling pathways were also identified. The top 10 items from different categories are presented in Figs. 4 and 5. The results indicated that cardiac muscle cell differentiation and glucose metabolic process items in $\mathrm{BP}$, the perinuclear region of cytoplasm and cytoplasmic microtubule items in CC, protein binding and double-stranded RNA binding items in MF, mucin type O-glycan biosynthesis and epithelial cell signaling in Helicobacter pylori infection items of the KEGG signaling pathways were the most closely associated with the target genes of miRNA-122.

\section{Discussion}

Although research regarding the expression levels and associated functions of miRNA-122 in HCC and other liver diseases have been widely carried out $(15,28)$, the reports are diffuse and contradictory. For example, miRNA-122 acted as a cancer suppressor gene and was downregulated in HCC tissues and cells compared with the adjacent normal tissues and normal cell lines $(15,29)$, which was in line with the results of TCGA analysis. Conversely, the serum expression levels of miRNA-122 increased significantly in HCC patients, hepatitis $\mathrm{C}$ patients and other liver injury diseases compared with the healthy controls $(30,31)$. From this perspective, miRNA-122 may be considered an onco-miRNA. In addition, the expression and function of miRNA-122 in other tumors have also been widely reported, including glioma (32), renal cell carcinoma (33), gastric (34) and pancreatic cancer (35) and others. In addition to cancer and liver injury diseases, miRNA-122 also served important roles in cardiovascular diseases (36-38), obesity (39-41), diabetes (42), immunological diseases (43-45) and other non-cancer diseases (46).

The present study analyzed the expression levels of miRNA-122 in tissues and serum samples of various types of cancer systematically by RT-qPCR analysis, TCGA and literature data. The lower expression levels of miRNA-122 in HCC tissues, compared with the adjacent normal tissues, had been validated by a number of researchers over the years $(47,48)$; however, one study has reported conflicting conclusions (49). The expression of miRNA-122 was multifarious in different types and degrees of liver cancer and liver injury diseases. From a large classification perspective, the expression levels of miRNA-122 were significantly increased in the serum of HCC patients compared with in healthy controls $(50,51)$. The expression levels of miRNA-122 in the serum of HBV-positive patients with HCC were frequently decreased compared with patients with benign liver lesions (27), which was consistent with the results of serological analysis in the present study. Meanwhile, miRNA-122 and AFP analyses were combined for diagnosis in the present study, which may have improved the non-invasive diagnostic accuracy of HCC.

TCGA database is one of the most widely used public data platforms for a variety of types of cancer. Following screening, 17 common cancer types were included in the differential expression analysis of miRNA-122. Finally, it was demonstrated that miRNA-122 was differentially expressed in 10 types of tumor from TCGA. However, by summarizing the reported literature, differential expression of miRNA-122 in a number of types of cancer was also demonstrated. Additionally, there are several consistent types of cancer between the two summary methods, including HCC (52), renal carcinoma (33) and colorectal cancer (53); however, certain results were inconsistent. The number of samples, fixed tumor type in TCGA, the difference in detection methods and platforms, etiology or pathological classification, and clinical features, may lead to inconsistent results. In future studies, an in-depth exploration of inconsistencies can also be conducted. In addition, the prognostic value of miRNA-122 in a variety of cancer was also analyzed and summarized. Through the analysis of TCGA data, it was demonstrated that the expression levels of miRNA-122 could affect the overall survival rate of HCC and renal carcinoma. In addition to the association between miRNA-122 and the prognosis of HCC, it was also demonstrated that the low expression levels of miRNA-122 predicted the poor prognosis of glioma (54), gastric cancer (55) and leukemia (56) by literature retrieval. However, there were disagreements regarding the association between the expression levels of miRNA-122 and the prognosis of HCC in the serum or plasma. Specific studies revealed that high expression of miRNA-122 indicated poor prognosis $(57,58)$, but certain findings suggested that the high expression of miRNA-122 predicted improved prognosis $(59,60)$; however, further investigation is required. Although there are a number of reports on miRNA-122, most of them are limited to a certain cancer, especially liver cancer. In the present study, a more comprehensive pan-cancer analysis of miRNA-122 was performed in combination with the current popular TCGA high-throughput sequencing database and associated literature. The present study is more general, gives a summary and can provide a macro guidance for the diagnosis, prognosis and treatment of multiple cancers in clinical practice.

In this study, the target genes of miRNA-122 were predicted using numerous platforms and the GO and KEGG enrichment analyses were performed on the reported target genes. The results of GO enrichment analysis demonstrated that the target genes of miRNA-122 may contribute to the composition of 
A
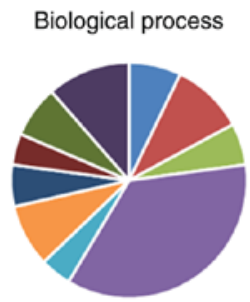

- Cardiac muscle cell differentiation

- Glucose metabolic process

" Magnesium ion transport

- Negative regulation of transcription from RNA polymerase II promoter

- Protein localization to chromatin

- O-glycan processing

- Cellular response to fatty acid

- Production of miRNAs involved in gene silencing by miRNA

- JNK cascade

- Negative regulation of gene expression

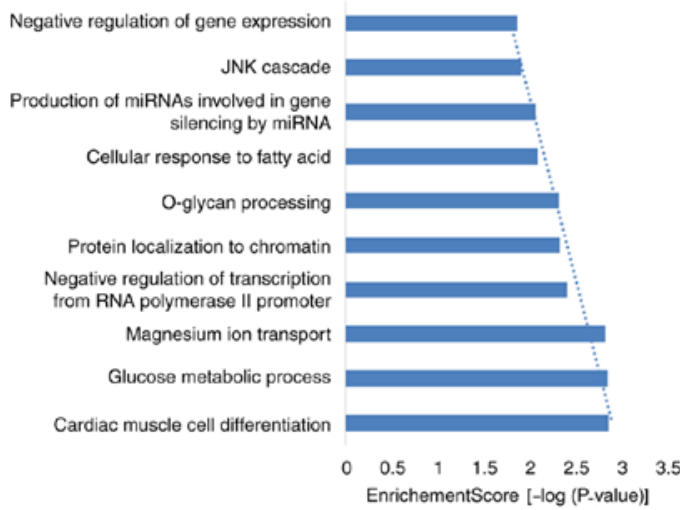

C

Molecular function

" Protein binding

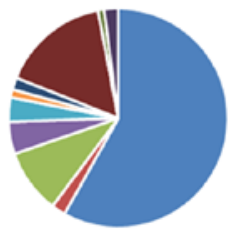

" Double-stranded RNA binding

" Transcription factor activity, sequence-specific DNA binding

- RNA polymerase II core promoter proximal region sequence-specific DNA binding

- Transcriptional activator activity, RNA polymerase II core promoter proximal

region sequence-specific binding

Polypeptide $\mathrm{N}$-acetylgalactosaminyitransterase activity

- RNA polymerase II distal enhancer sequence-specific DNA binding

" Metal ion binding

" Cobalamin binding

- Histone deacetylase binding

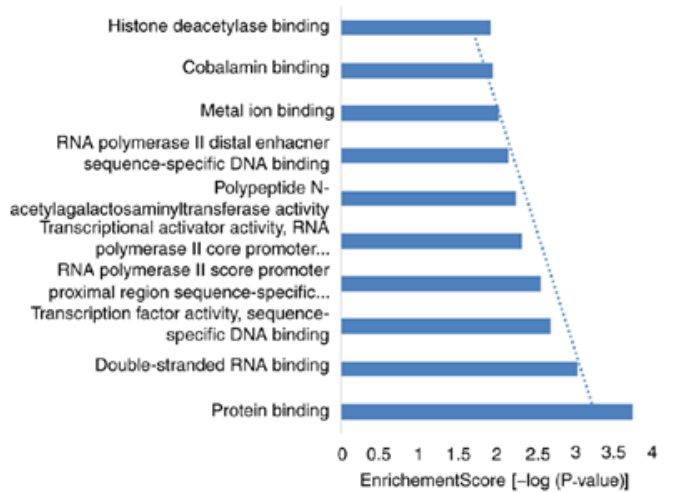

B

Cellular component

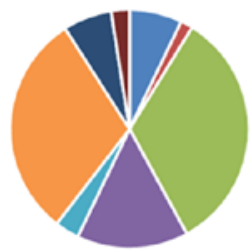

- Perinuclear region of cytoplasm

" Cytoplasmic microtubule

- Nucleus

- Membrane

- Cell-cell adherens junction

- Cytoplasm

- Golgi apparatus

- Actin cytoskeleton

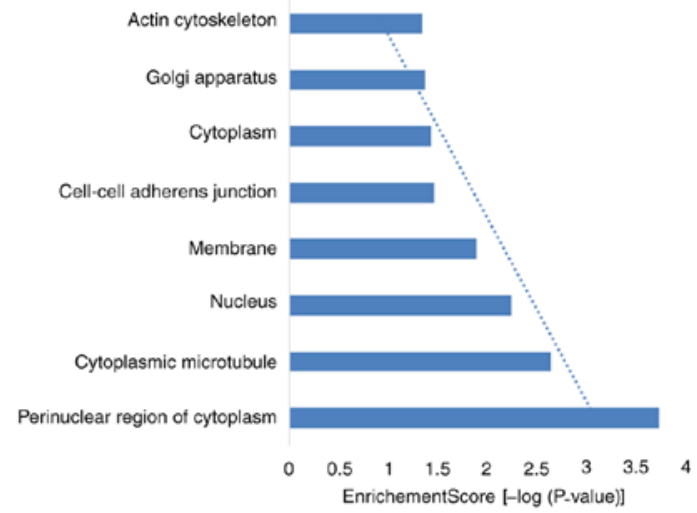




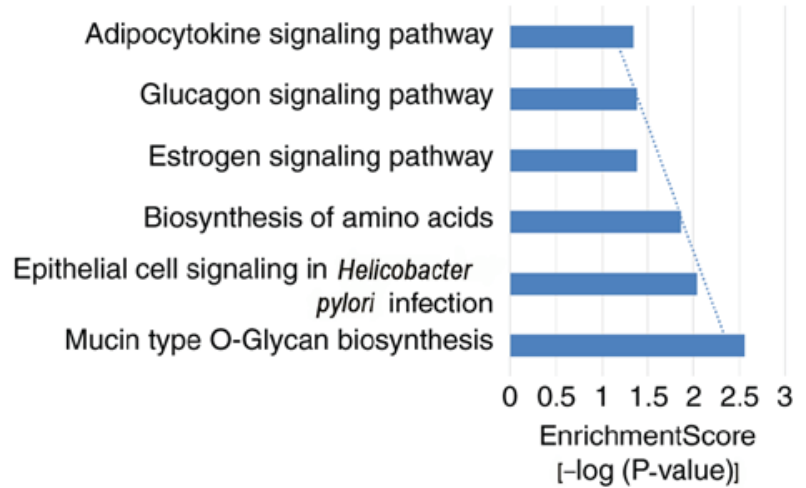

Figure 5. Kyoto Encyclopedia of Genes and Genomes pathway enrichment analysis of miRNA-122 target genes by Database for Annotation, Visualization and Integrated Discovery software.

the nucleus and cytoplasm, and regulate cardiac muscle cell differentiation in a variety of biological processes, including cardiac muscle cell differentiation and glucose metabolic processes through protein binding. So far, numerous articles have reported that miRNA-122 was closely associated with heart-related diseases, obesity and diabetes $(37,61,62,64)$. For example, the expression of miR-122 was involved in hypoxia-induced cardiomyocyte injury (37). In experiments with miniature pigs, researchers demonstrated that increases in weight and cholesterol levels could lead to a decline in the expression levels of miRNA-122, which supported the association of miRNA122 in obesity (61). KEGG pathway analysis revealed that the target genes were mainly enriched in protein biosynthesis, estrogen and glucagon associated signaling pathways. Munagala et al (62) reported that miRNA-122 was one of the significantly modulated miRNAs in the process of estrogen-mediated mammary carcinogenesis. miRNA-122 participated in the regulation of insulin resistance, diabetes and metabolic syndrome, which were all likely to be associated with glucagon related signaling pathways (42).

In conclusion, the clinical significance of miRNA-122 was systematically analyzed from multiple perspectives in this study. The diagnostic value of miRNA-122 between the HCC and healthy control via serology was further demonstrated, and the differential diagnostic value in patients with HCC compared with in benign lesions was also analyzed. In addition, it was demonstrated that miRNA-122 exhibited significant differential expression and prognostic ability in various types of cancer via TCGA database and literature review. Finally, a number of important biological functions and regulatory mechanisms have been reported through the enrichment analysis of the miRNA-122 target genes. These analyses demonstrated that miRNA-122 may be an indispensable biomarker for the diagnosis, prognostic evaluation and targeted therapy in a variety of cancer types.

\section{Acknowledgements}

The authors would like to thank Mrs Yujie Huang (Department of Clinical Laboratory, First Affiliated Hospital of Guangxi Medical University, Guangxi, China) for her work in language editing.

\section{Funding}

No funding was received.

\section{Availability of data and materials}

The datasets generated and/or analyzed during the current study are available in the TCGA repository (https://cancergenome.nih.gov/).

\section{Authors' contributions}

MD contributed to the writing of the paper and bioinformatics analysis; LL contributed to the sample collection and experiment; XQ contributed to the overall design of this paper.

\section{Ethics approval and consent to participate}

The present study was approved by the Institutional Review Board of the First Affiliated Hospital of Guangxi Medical University and written informed consent was obtained from all individuals in this research project.

\section{Patient consent for publication}

All the patients have written informed consent for the publication of any associated data or accompanying images.

\section{Competing interests}

The authors declare they have no competing interests.

\section{References}

1. Forner A, Llovet JM and Bruix J: Hepatocellular carcinoma. Lancet 379: 1245-1255, 2012.

2. Yang X, Xie X, Xiao YF, Xie R, Hu CJ, Tang B,Li BS and Yang SM: The emergence of long non-coding RNAs in the tumorigenesis of hepatocellular carcinoma. Cancer Lett 360: 119-124, 2015.

3. El-Serag HB: Epidemiology of viral hepatitis and hepatocellular carcinoma. Gastroenterology 142: 1264-1273 e1, 2012.

4. Liu CJ and Kao JH: Global perspective on the natural history of chronic hepatitis B: role of hepatitis B virus genotypes A to J. Semin Liver Dis 33: 97-102, 2013.

5. Fabian MR, Sonenberg N and Filipowicz W: Regulation of mRNA translation and stability by microRNAs. Annu Rev Biochem 79: 351-379, 2010.

6. Fukaya T and Tomari Y: MicroRNAs mediate gene silencing via multiple different pathways in drosophila. Molecular cell 48: 825-836, 2012.

7. Takasaki S: Roles of microRNAs in cancers and development. Methods Mol Biol 1218: 375-413, 2015.

8. Zhang LF, Jiang S and Liu MF: MicroRNA regulation and analytical methods in cancer cell metabolism. Cell Mol Life Sci 74: 2929-2941, 2017.

9. Castro D, Moreira M, Gouveia AM, Pozza DH and De Mello RA: MicroRNAs in lung cancer. Oncotarget 8: 81679-81685, 2017.

10. Ferracin M, Lupini L, Mangolini A and Negrini M: Circulating non-coding RNA as biomarkers in colorectal cancer. Adv Exp Med Biol 937: 171-181, 2016.

11. Endzeliņš E, Melne V, Kalnina Z, Lietuvietis V, Riekstiņa U, Llorente A and Linē A: Diagnostic, prognostic and predictive value of cell-free miRNAs in prostate cancer: A systematic review. Mol Cancer 15: 41, 2016.

12. Bandiera S, Pfeffer S, Baumert TF and Zeisel MB: miR-122-a key factor and therapeutic target in liver disease. J Hepatol 62: 448-457, 2015.

13. Wen J and Friedman JR: miR-122 regulates hepatic lipid metabolism and tumor suppression. J Clin Invest 122: 2773-2776, 2012. 
14. Gao Y, He Y, Ding J, Wu K, Hu B, Liu Y, Wu Y, Guo B, Shen Y, Landi D, et al: An insertion/deletion polymorphism at miRNA-122-binding site in the interleukin-1alpha 3' untranslated region confers risk for hepatocellular carcinoma. Carcinogenesis 30: 2064-2069, 2009.

15. Ma J, Li T, Han X and Yuan H: Knockdown of LncRNA ANRIL suppresses cell proliferation, metastasis, and invasion via regulating miR-122-5p expression in hepatocellular carcinoma. J Cancer Res Clin Oncol 144: 205-214, 2018.

16. Caviglia GP, Abate ML, Gaia S, Petrini E, Bosco C, Olivero A, Rosso C, Ciancio A, Pellicano R, Saracco GM, et al: Risk of hepatocellular carcinoma in HBV cirrhotic patients assessed by the combination of miR-122, AFP and PIVKA-II. Panminerva Med 59: 283-289, 2017.

17. Ding Y, Yan JL, Fang AN, Zhou WF and Huang L: Circulating miRNAs as novel diagnostic biomarkers in hepatocellular carcinoma detection: A meta-analysis based on 24 articles. Oncotarget 8: 66402-66413, 2017.

18. NaderiM,Pazouki A,ArefianE,HashemiSM,Jamshidi-AdeganiF, Gholamalamdari O, Soudi S, Azadmanesh K, Mirab Samiee S, Merat S, et al: Two triacylglycerol pathway genes, CTDNEP1 and LPIN1, are down-regulated by hsa-miR-122-5p in hepatocytes. Arch Iran Med 20: 165-171, 2017.

19. Schisterman EF, Perkins NJ, Liu A and Bondell H: Optimal cut-point and its corresponding Youden Index to discriminate individuals using pooled blood samples. Epidemiology 16: 73-81, 2005 .

20. Chen X, Dai M, Zhu H, Li J, Huang Z, Liu X, Huang Y, Chen J and Dai S: Evaluation on the diagnostic and prognostic values of long non-coding RNA BLACAT1 in common types of human cancer. Mol Cancer 16: 160, 2017.

21. Goldvaser H, Gutkin A, Beery E, Edel Y, Nordenberg J, Wolach O, Rabizadeh E, Uziel O and Lahav M: Characterisation of blood-derived exosomal hTERT mRNA secretion in cancer patients: A potential pan-cancer marker. Br J Cancer 117: 353-357, 2017.

22. Betel D, Wilson M, Gabow A, Marks DS and Sander C: The microRNA.org resource: Targets and expression. Nucleic Acids Res 36 (Database Issue): D149-D153, 2008.

23. Krek A, Grün D, Poy MN, Wolf R, Rosenberg L, Epstein EJ, MacMenamin P, da Piedade I, Gunsalus KC, Stoffel M and Rajewsky N: Combinatorial microRNA target predictions. Nat Genet 37: 495-500, 2005.

24. Wong N and Wang X: miRDB: An online resource for microRNA target prediction and functional annotations. Nucleic Acids Res 43 (Database Issue): D146-D152, 2015.

25. Agarwal V, Bell GW, Nam JW and Bartel DP: Predicting effective microRNA target sites in mammalian mRNAs. Elife 4, 2015

26. Huang da W, Sherman BT and Lempicki RA: Bioinformatics enrichment tools: Paths toward the comprehensive functional analysis of large gene lists. Nucleic Acids Res 37: 1-13, 2009.

27. Qiao DD, Yang J, Lei XF, Mi GL, Li SL, Li K, Xu CQ and Yang HL: Expression of microRNA-122 and microRNA-22 in HBV-related liver cancer and the correlation with clinical features. Eur Rev Med Pharmacol Sci 21: 742-747, 2017.

28. Wang Y, Zhu P, Qiu J, Wang J, Zhu H, Zhu Y, Zhang L, Zhu J, Liu X and Dong C: Identification and characterization of interferon signaling-related microRNAs in occult hepatitis B virus infection. Clin Epigenetics 9: 101, 2017.

29. Dai R, Peng F, Xiao X, Gong X, Jiang Y, Zhang M, Tian Y, Xu Y, Ma J, Li M, et al: Hepatitis B virus X protein-induced upregulation of CAT-1 stimulates proliferation and inhibits apoptosis in hepatocellular carcinoma cells. Oncotarget 8: 60962-60974, 2017.

30. Ali HEA, Abdel Hameed R, Effat H, Ahmed EK, Atef AA, Sharawi SK, Ali M, Abd Elmageed ZY and Abdel Wahab AH: Circulating microRNAs panel as a diagnostic tool for discrimination of HCV-associated hepatocellular carcinoma. Clin Res Hepatol Gastroenterol 41: e51-e62, 2017.

31. Murray DD, Suzuki K, Law M, Trebicka J, Neuhaus Nordwall J, Johnson M, Vjecha MJ, Kelleher AD and Emery S: Circulating miR-122 and miR-200a as biomarkers for fatal liver disease in ART-treated, HIV-1-infected individuals. Sci Rep 7: 10934, 2017.

32. Su R, Cao S, Ma J, Liu Y, Liu X, Zheng J, Chen J, Liu L, Cai H, $\mathrm{Li}$ Z, et al: Knockdown of SOX2OT inhibits the malignant biological behaviors of glioblastoma stem cells via up-regulating the expression of miR-194-5p and miR-122. Mol Cancer 16: 171, 2017.

33. Fan Y, Ma X, Li H, Gao Y, Huang Q, Zhang Y, Bao X, Du Q, Luo G, Liu K, et al: miR-122 promotes metastasis of clear-cell renal cell carcinoma by downregulating Dicer. Int J Cancer 142: 547-560, 2018
34. Rao M, Zhu Y, Zhou Y, Cong X and Feng L: MicroRNA-122 inhibits proliferation and invasion in gastric cancer by targeting CREB1. Am J Cancer Res 7: 323-333, 2017.

35. Calatayud D, Dehlendorff C, Boisen MK, Hasselby JP, Schultz NA, Werner J, Immervoll H, Molven A, Hansen CP and Johansen JS: Tissue MicroRNA profiles as diagnostic and prognostic biomarkers in patients with resectable pancreatic ductal adenocarcinoma and periampullary cancers. Biomark Res 5: 8, 2017.

36. Li XD, Yang YJ, Wang LY, Qiao SB, Lu XF, Wu YJ, Xu B, Li HF and Gu DF: Elevated plasma miRNA-122, -140-3p, -720, -2861, and -3149 during early period of acute coronary syndrome are derived from peripheral blood mononuclear cells. PLoS One 12: e0184256, 2017.

37. Zhang Z, Li H, Chen S, Li Y, Cui Z and Ma J: Knockdown of MicroRNA-122 protects H9c2 cardiomyocytes from hypoxia-induced apoptosis and promotes autophagy. Med Sci Monit 23: 4284-4290, 2017.

38. Satrauskienè A, Navickas R, Laucevičius A and Huber HJ: Identifying differential miR and gene consensus patterns in peripheral blood of patients with cardiovascular diseases from literature data. BMC Cardiovasc Disord 17: 173, 2017.

39. Blum A, Yehuda H, Geron N and Meerson A: Elevated levels of miR-122 in serum may contribute to improved endothelial function and lower oncologic risk following bariatric surgery. Isr Med Assoc J 19: 620-624, 2017.

40. Jones A, Danielson KM, Benton MC, Ziegler O, Shah R, Stubbs RS, Das S and Macartney-Coxson D: miRNA signatures of insulin resistance in obesity. Obesity (Silver Spring) 25: $1734-1744,2017$

41. Zhao H, Shen J, Daniel-MacDougall C, Wu X and Chow WH: Plasma MicroRNA signature predicting weight gain among Mexican-American women. Obesity (Silver Spring) 25: 958-964, 2017.

42. Willeit P, Skroblin P, Moschen AR, Yin X, Kaudewitz D, Zampetaki A, Barwari T, Whitehead M, Ramírez CM, Goedeke L, et al: Circulating MicroRNA-122 is associated with the risk of new-onset metabolic syndrome and type 2 diabetes. Diabetes 66: 347-357, 2017

43. Bijkerk R, Florijn BW, Khairoun M, Duijs JMGJ, Ocak G, de Vries APJ, Schaapherder AF, Mallat MJK, de Fijter JW, Rabelink TJ, et al: Acute rejection after kidney transplantation associates with circulating MicroRNAs and vascular injury. Transplant Direct 3: e174, 2017.

44. Wang T, Li F, Geng W, Ruan Q and Shi W: MicroRNA-122 ameliorates corneal allograft rejection through the downregulation of its target CPEB1. Cell Death Discov 3: 17021, 2017.

45. Selmaj I, Cichalewska M, Namiecinska M, Galazka G, Horzelski W, Selmaj KW and Mycko MP: Global exosome transcriptome profiling reveals biomarkers for multiple sclerosis. Ann Neurol 81: 703-717, 2017.

46. Trzybulska D, Bobjer J, Giwercman A and Tsatsanis C: Serum microRNAs in male subfertility-biomarkers and a potential pathogenetic link to metabolic syndrome. J Assist Reprod Genet 34: 1277-1282, 2017.

47. Coulouarn C, Factor VM, Andersen JB, Durkin ME and Thorgeirsson SS: Loss of miR-122 expression in liver cancer correlates with suppression of the hepatic phenotype and gain of metastatic properties. Oncogene 28: 3526-3536, 2009.

48. Jin Y, Wang J, Han J, Luo D and Sun Z: MiR-122 inhibits epithelial-mesenchymal transition in hepatocellular carcinoma by targeting Snaill and Snail2 and suppressing WNT/ $\beta$-cadherin signaling pathway. Exp Cell Res 360: 210-217, 2017.

49. Miquelestorena-Standley E, Tallet A, Collin C, Piver E, De Muret A, Salamé E, Bourlier P, Kervarrec T, Guyétant S and Pagès JC: Interest of variations in microRNA-152 and -122 in a series of hepatocellular carcinomas related to hepatitis $\mathrm{C}$ virus infection. Hepatol Res 48: 566-573, 2018.

50. Hung CH, Hu TH, Lu SN, Kuo FY, Chen $\mathrm{CH}$, Wang JH, Huang CM, Lee CM, Lin CY, Yen YH and Chiu YC: Circulating microRNAs as biomarkers for diagnosis of early hepatocellular carcinoma associated with hepatitis B virus. Int J Cancer 138: 714-720, 2016

51. El-Garem H, Ammer A, Shehab H, Shaker O, Anwer M, El-Akel W and Omar H: Circulating microRNA, miR-122 and miR-221 signature in Egyptian patients with chronic hepatitis $\mathrm{C}$ related hepatocellular carcinoma. World J Hepatol 6: 818-824, 2014.

52. Xu Q, Zhang M, Tu J, Pang L, Cai W and Liu X: MicroRNA-122 affects cell aggressiveness and apoptosis by targeting PKM2 in human hepatocellular carcinoma. Oncol Rep 34: 2054-2064, 2015. 
53. Kanaan Z, Rai SN, Eichenberger MR, Barnes C, Dworkin AM, Weller C, Cohen E, Roberts H, Keskey B, Petras RE, et al: Differential microRNA expression tracks neoplastic progression in inflammatory bowel disease-associated colorectal cancer. Hum Mutat 33: 551-560, 2012.

54. Tang Y, Zhao S, Wang J, Li D, Ren Q and Tang Y: Plasma miR-122 as a potential diagnostic and prognostic indicator in human glioma. Neurol Sci 38: 1087-1092, 2017.

55. Chen Q, Ge X, Zhang Y, Xia H, Yuan D, Tang Q, Chen L, Pang X, Leng W and Bi F: Plasma miR-122 and miR-192 as potential novel biomarkers for the early detection of distant metastasis of gastric cancer. Oncol Rep 31: 1863-1870, 2014.

56. Yang J, Yuan Y, Yang X, Hong Z and Yang L: Decreased expression of microRNA-122 is associated with an unfavorable prognosis in childhood acute myeloid leukemia and function analysis indicates a therapeutic potential. Pathol Res Pract 213: 1166-1172, 2017

57. Cho HJ, Kim JK, Nam JS, Wang HJ, Lee JH, Kim BW, Kim SS, Noh CK, Shin SJ, Lee KM, et al: High circulating microRNA-122 expression is a poor prognostic marker in patients with hepatitis $\mathrm{B}$ virus-related hepatocellular carcinoma who undergo radiofrequency ablation. Clin Biochem 48: 1073-1078, 2015.

58. Liu M, Liu J, Wang L, Wu H, Zhou C, Zhu H, Xu N and Xie Y: Association of serum microRNA expression in hepatocellular carcinomas treated with transarterial chemoembolization and patient survival. PLoS One 9: e109347, 2014.

59. Xu Y, Bu X, Dai C and Shang C: High serum microRNA-122 level is independently associated with higher overall survival rate in hepatocellular carcinoma patients. Tumour Biol 36 4773-4776, 2015

60. Köberle V, Kronenberger B, Pleli T, Trojan J, Imelmann E, Peveling-Oberhag J, Welker MW, Elhendawy M, Zeuzem S, Piiper A and Waidmann O: Serum microRNA-1 and microRNA-122 are prognostic markers in patients with hepatocellular carcinoma. Eur J Cancer 49: 3442-3449, 2013

61. Cirera S, Birck M, Busk PK and Fredholm M: Expression profiles of miRNA-122 and its target CAT1 in minipigs (Sus scrofa) fed a high-cholesterol diet. Comp Med 60: 136-141, 2010.

62. Munagala R, Aqil F, Vadhanam MV and Gupta RC: MicroRNA 'signature' during estrogen-mediated mammary carcinogenesis and its reversal by ellagic acid intervention. Cancer Lett 339: 175-184, 2013.

63. El-Abd NE, Fawzy NA, El-Sheikh SM and Soliman ME: Circulating miRNA-122, miRNA-199a, and miRNA-16 as biomarkers for early detection of hepatocellular carcinoma in egyptian patients with chronic hepatitis $\mathrm{C}$ virus infection. Mol Diagn Ther 19: 213-220, 2015.

64. Wang G, Zhao Y and Zheng Y: MiR-122/Wnt/ $\beta$-catenin regulatory circuitry sustains glioma progression. Tumour Biol 35 8565-8572, 2014.

65. Khare D, Goldschmidt N, Bardugo A, Gur-Wahnon D, Ben-Dov IZ and Avni B: Plasma microRNA profiling: Exploring better biomarkers for lymphoma surveillance. PLoS One 12: e0187722, 2017.

66. Conceição AL, Da Silva CT, Badial RM, Valsechi MC, Stuqui B, Goncalves JD, Jasiulionis MG, De Freitas Calmon M and Rahal P: Downregulation of OCLN and GAS1 in clear cell renal cell carcinoma. Oncol Rep 37: 1487-1496, 2017.

67. Nientiedt M, Deng M, Schmidt D, Perner S, Müller SC and Ellinger J: Identification of aberrant tRNA-halves expression patterns in clear cell renal cell carcinoma. Sci Rep 6: 37158, 2016

68. Jingushi K, Kashiwagi Y, Ueda Y, Kitae K, Hase H, Nakata W, Fujita K, Uemura M, Nonomura $\mathrm{N}$ and Tsujikawa K: High miR-122 expression promotes malignant phenotypes in ccRCC by targeting occludin. Int J Oncol 51: 289-297, 2017.

69. Munari E, Marchionni L, Chitre A, Hayashi M, Martignoni G, Brunelli M, Gobbo S, Argani P, Allaf M, Hoque MO and Netto GJ: Clear cell papillary renal cell carcinoma: micro-RNA expression profiling and comparison with clear cell renal cell carcinoma and papillary renal cell carcinoma. Hum Pathol 45: $1130-1138,2014$
70. Wang Z, Qin C, Zhang J, Han Z, Tao J, Cao Q, Zhou W, Xu Z, Zhao C, Tan $R$ and Gu M: MiR-122 promotes renal cancer cell proliferation by targeting Sprouty2. Tumour Biol 39: 1010428317691184,2017

71. Wotschofsky Z, Busch J, Jung M, Kempkensteffen C, Weikert S, Schaser KD, Melcher I, Kilic E, Miller K, Kristiansen G, et al: Diagnostic and prognostic potential of differentially expressed miRNAs between metastatic and non-metastatic renal cell carcinoma at the time of nephrectomy. Clin Chim Acta 416: 5-10, 2013.

72. Papaconstantinou IG, Manta A, Gazouli M, Lyberopoulou A, Lykoudis PM, Polymeneas G and Voros D: Expression of microRNAs in patients with pancreatic cancer and its prognostic significance. Pancreas 42: 67-71, 2013.

73. Schultz NA, Dehlendorff C, Jensen BV, Bjerregaard JK, Nielsen KR, Bojesen SE, Calatayud D, Nielsen SE, Yilmaz M, Holländer NH, et al: MicroRNA biomarkers in whole blood for detection of pancreatic cancer. JAMA 311: 392-404, 2014

74. Carter JV, Roberts HL, Pan J, Rice JD, Burton JF, Galbraith NJ, Eichenberger MR, Jorden J, Deveaux P, Farmer R, et al: A highly predictive model for diagnosis of colorectal neoplasms using plasma MicroRNA: Improving specificity and sensitivity. Ann Surg 264: 575-584, 2016.

75. Liu N, Jiang F, He TL, Zhang JK, Zhao J, Wang C, Jiang GX, Cao LP, Kang PC, Zhong XY, et al: The roles of MicroRNA-122 overexpression in inhibiting proliferation and invasion and stimulating apoptosis of human cholangiocarcinoma cells. Sci Rep 5: 16566, 2015.

76. Lu W, Zhang Y, Zhou L, Wang X, Mu J, Jiang L, Hu Y, Dong P and Liu Y: miR-122 inhibits cancer cell malignancy by targeting PKM2 in gallbladder carcinoma. Tumour Biol, Nov 6, 2015 (Epub ahead of print).

77. Walter BA, Valera VA, Pinto PA and Merino MJ: Comprehensive microRNA profiling of prostate cancer. J Cancer 4: 350-357, 2013.

78. Venkatesan N, Deepa PR, Khetan V and Krishnakumar S: Computational and in vitro investigation of miRNA-Gene regulations in retinoblastoma pathogenesis: miRNA mimics strategy. Bioinform Biol Insights 9: 89-101, 2015.

79. Xiao F, Chen J, Lian C, Han P and Zhang C: Tumor necrosis factor-related apoptosis-inducing ligand induces cytotoxicity specific to osteosarcoma by microRNA response elements. Mol Med Rep 11: 739-745, 2015

80. Ergün S, Ulasli M, Igci YZ, Igci M, Kırkbes S, Borazan E, Balik A, Yumrutas Ö, Camci C, Cakmak EA, et al: The association of the expression of miR-122-5p and its target ADAM10 with human breast cancer. Mol Biol Rep 42: 497-505, 2015.

81. Yan Y, Zhang F, Fan Q, Li X and Zhou K: Breast cancer-specific TRAIL expression mediated by miRNA response elements of let-7 and miR-122. Neoplasma 61: 672-679, 2014.

82. Wang B, Wang H and Yang Z: MiR-122 inhibits cell proliferation and tumorigenesis of breast cancer by targeting IGF1R. PLoS One 7: e47053, 2012

83. Peng Y, Li C, Luo DC, Ding JW, Zhang W and Pan G: Expression profile and clinical significance of microRNAs in papillary thyroid carcinoma. Molecules 19: 11586-11599, 2014.

84. Stilling G, Sun Z, Zhang S, Jin L, Righi A, Kovāes G, Korbonits M, Scheithauer BW, Kovacs K and Lloyd RV: MicroRNA expression in ACTH-producing pituitary tumors: Up-regulation of microRNA-122 and -493 in pituitary carcinomas. Endocrine 38: $67-75,2010$

85. Wang Y, Xing QF, Liu XQ, Guo ZJ, Li CY and Sun G: MiR-122 targets VEGFC in bladder cancer to inhibit tumor growth and angiogenesis. Am J Transl Res 8: 3056-3066, 2016.

This work is licensed under a Creative Commons Attribution-NonCommercial-NoDerivatives 4.0 International (CC BY-NC-ND 4.0) License. 